\section{Loss of Weight and Metabolic Rate of Rats with Lesions in the Medial and Lateral Hypothalamus}

RATs with large bilateral lesions of the lateral hypothalamus refuse to eat or drink and, unless force-fed and watered, die within two weeks of operation ${ }^{1-3}$. During an examination of these animals ${ }^{3}$ we noticed that the rate of lose of body-weight was greater and the duration of survival shorter than in intact rats deprived of food and water. Morgane ${ }^{4}$ has also remarked on this more rapid deterioration and has suggested that such lesions cause a specific 'metabolic decay'.

The oxygen consumption of : (1) aphagic-adipsic rats ; (2) rats with hypothalamic hyperphagia from ventromedial destruction previously starved to a similar weight ; (3) control rats, was measured under standard conditions. Groups 2 and 3 did not receive food or water in this experiment so that they would be under the same nutritional condition as the first group. The resting oxygen consumption and metabolic rate of the normal and hypothalamic hyperphagic rats were almost identical when the body-weights were comparable (Table 1). However, the mean oxygen consumption and metabolic rate of rats with aphagia and adipsia were significantly greater than those of the other two groups.

Table 1. Metabolio Rate of Rats with Lateral Hypothatamo Lesions AND OF NORMAI AND PREVIOUSLY OBESE STARVED RATS

\begin{tabular}{|c|c|c|c|}
\hline & No. & $\begin{array}{l}\text { Body-weight } \\
(\mathrm{g})\end{array}$ & $\begin{array}{l}\text { Metabolic rate } \\
\text { ml. } \mathrm{O}_{2} / \mathrm{min} \\
\mathrm{kg}^{2 / 4}\end{array}$ \\
\hline Normal (starved) & 9 & $188.4 \pm 3 \cdot 7^{*}$ & $9.97 \pm 0.52$ \\
\hline Lateral lesioned & 17 & $183 \cdot 9 \pm 4 \cdot 7$ & $\begin{array}{c}<0.01 \\
13.75 \pm 0.83\end{array}$ \\
\hline $\begin{array}{l}\text { Previously obese (starved) } \\
\quad * \text { Mean } \pm \text { S.E. }\end{array}$ & 11 & $182 \cdot 5 \pm 2 \cdot 6$ & $0.58 \pm 0.54$ \\
\hline
\end{tabular}

The inmediate effect of hypothalamic lesions on the metabolic rate was determined in another experiment which consisted of four rats with hyperphagia-producing lesions in the ventromedial region of the hypothalamus, six rats with lesions in the lateral hypothalamus containing the 'feeding' and 'drinking' systems, and four shamoperated controls. To provide precise controls, the rats with obesity-producing lesions and the sham-operated controls were not given food or water post-operatively. The rats with lateral lesions were provided with a high-fat diet and water ad libitum, but they did not eat or drink. Resting metabolic rates were determined before and at $4,24,48$, and $72 \mathrm{~h}$ after operation. The loss of body-weight of both lesioned groups of rats was greater than that of the sham-operated control rats (Fig. 1). The metabolic rate of the rats with either lesion increased by approximately 50 per cent within the first $4 \mathrm{~h}$ and gradually returned to pre-operative levels by the third day (Fig. 2). The meta-

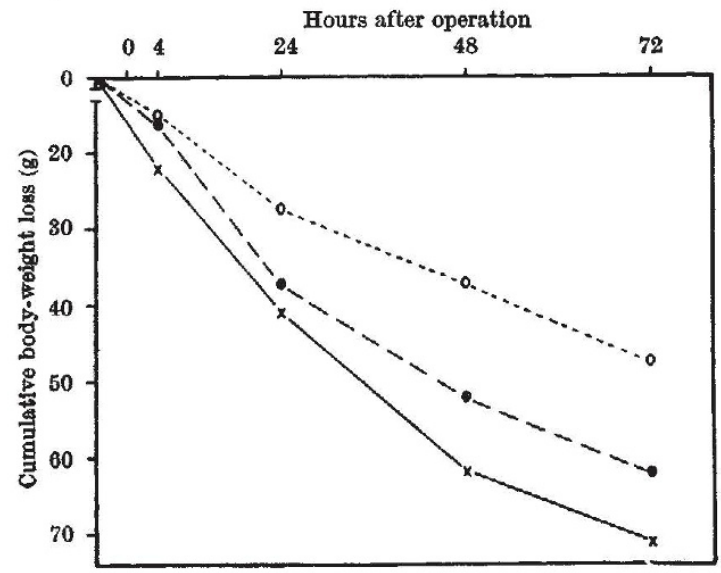

Fig. 1. Changes in body-weight during the first three days after bilateral electrolytic ablation in the lateral or ventromedial region of the hypothalamus. 'The 'ventromedial' and control rats were deprived of rood and water from which the 'lateral' rats abstained spontaneously;

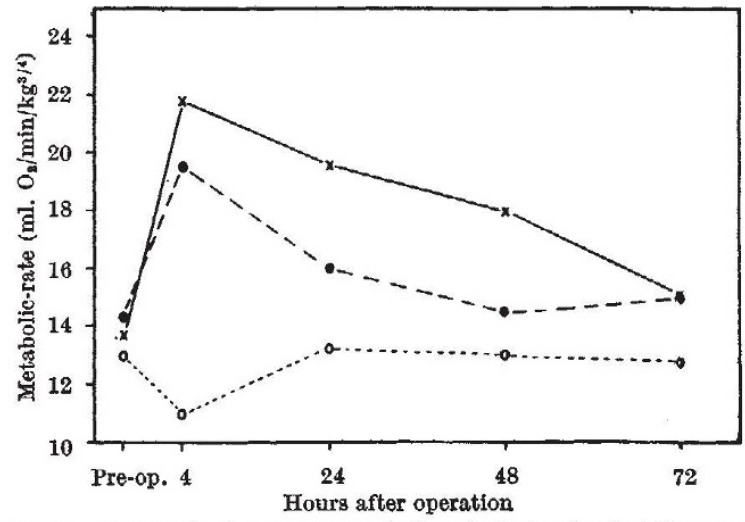

Fig. 2. Changes in the resting metabolic rate during the flrst three days after bilateral electrolytic ablation in the lateral or ventromedial region of the hypothalamus. 'The 'ventromedial' and control rats were deprived $x$, Medial lesions (4); O, lateral lesions $(6) ; O$, operated controls (4)

bolic rate of the sham-operated control rats fell within the first $4 \mathrm{~h}$ after operation, returned to normal by $24 \mathrm{~h}$ and then showed the gradual decline associated with starvation. The relative increase in metabolism of the rats with medial or lateral lesions during the first three days after operation may be sufficient to account for their more rapid weight loss and earlier death. It is known that the metabolic rate of the hypothalamic-obese rat rises after the operation; but this catabolic tendency is overshadowed by their tremendous and usually immediate hyperphagia. When food is not available the post-operative hypermetabolism leads to an abnormally rapid loss in weight similar to that seen in rats that spontaneously refuse food and water following lesions in the lateral hypothalamus.

It therefore seems quite likely that the rapid loss in weight and consequent early death of the rats with lesions in the 'feeding' and 'drinking' systems of the lateral hypothalamus are attributable to their relatively high metabolic rate in the face of starvation and not to a specific effect.

This work was supported by grants from the National Research Council of Canada and the Defence Research Board of Canada.

J. A. F. STEVENSON

D. G. MontemurRo

Department of Physiology,

University of Western Ontario, London, Ontario.

${ }^{1}$ Anand, B. K., and Brobeck, J. R., Yale J. Biol. Med., 24, 123 (1951).

${ }^{2}$ Anand, B. K., and Brobeck, J. R., Proc. Soc. Exp. Biol. and Med., 77, 323 (1951).

Montemurro, D. G, thesis, Univ, Western Ontario (1957).

- Morgane, P. J., J. Comp. Neurol., 117, 1 (1961).

\section{Effect of Alcohol on Release of Oxytocin}

IT is known from the work of van Dyke and Ames" that previously administered ethanol blocks the release of antidiuretic hormone (ADH) in response to acetylcholine. Furthermore, during ethanol anæsthesia antidiuretic activity can be detected neither in the blood from the head nor in the urine, and injection of hypertonic saline into the carotid artery does not produce any significant antidiuretic response ${ }^{2}$. It was found of interest to investigate the effect of ethanol also on the pituitary release of oxytocin. Since assay methods for oxytocin in blood are difficult and rather insensitive, it was found best to study the effect on the puerperal uterus of oxytocin released by suckling in the milk let-down reflex. The post-partum uterus of the rabbit is extremely sensitive to oxytocin, the threshold dose on the first post-partum day, being about $0.03 \mathrm{mu}$, oxytocin intravenously per $\mathrm{kg}$ body-weight ${ }^{3}$. As is the case in the human, suckling of the young produces a strong oxytocic response on the uterus during the first days after delivery in the rabbit. 